\title{
Deep Learning-based Time-varying Channel Estimation for RIS Assisted Communication
}

\author{
Meng Xu, Shun Zhang, Senior Member, IEEE, Jianpeng Ma, Member, IEEE, Octavia A. Dobre, Fellow, IEEE
}

\begin{abstract}
Reconfigurable intelligent surface (RIS) is considered as a revolutionary technology for future wireless communication networks. In this letter, we consider the acquisition of the time-varying cascaded channels, which is a challenging task due to the massive number of passive RIS elements and the small channel coherence time. To reduce the pilot overhead, a deep learning-based channel extrapolation is implemented over both antenna and time domains. We divide the neural network into two parts, i.e., the time-domain and the antenna-domain extrapolation networks, where the neural ordinary differential equations (ODE) are utilized. In the former, ODE accurately describes the dynamics of the RIS channels and improves the recurrent neural network's performance of time series reconstruction. In the latter, ODE is resorted to modify the relations among different data layers in a feedforward neural network. We cascade the two networks and jointly train them. Simulation results show that the proposed scheme can effectively extrapolate the cascaded RIS channels in high mobility scenario.
\end{abstract}

Index Terms-Deep learning, RIS, channel extrapolation, ordinary differential equation, recurrent neural network.

\section{INTRODUCTION}

Massive and diversified communication services put forward higher requirements, such as low energy cost and full coverage, to the upcoming $6 \mathrm{G}$ communication system. As a promising new technology, reconfigurable intelligent surface (RIS) has attracted more and more attentions. With the artificial electromagnetic structure, RIS can actively customize the wireless propagation link. Specially, by applying control signals to the tunable elements on the electromagnetic units, the electromagnetic properties of these units can be controlled dynamically [1]. Moreover, RIS can work in the passive model, which can greatly decrease the system's power consumption. Recent studies demonstrate that RIS can improve the quality of the received signal, expand the coverage range and enhance the capacity of the wireless network [2] - [3].

In order to fully embrace the above advantages of RIS, accurate channel state information (CSI) should be acquired. In [4], Liu et al. proposed a message passing-based algorithm to factorize the cascaded channels. The authors in [5] adopted a two-stage channel estimation scheme by using atomic norm minimization to sequentially estimate the channel parameters. In [6], Kim et al. proposed a single-path approximated channel and selective emphasis on rank-one matrices to enable practical IRS-empowered SU-MIMO systems with low training

M. Xu, S. Zhang and J. Ma are with the State Key Laboratory of Integrated Services Networks, Xidian University, Xian 710071, P. R. China (e-mail: mxu_20@stu.xidian.edu.cn, zhangshunsdu@xidian.edu.cn,jpmaxdu@gmail.com).

O. A. Dobre is with Faculty of Engineering and Applied Science, Memorial University, St. John's NL A1B 3X5, Canada (e-mail: odobre@mun.ca). overhead. As mentioned in [4] - [6], the main challenge for the channel estimation over RIS networks comes from the large number of passive reflection elements at the RIS node. In order to decrease the channel estimation overhead, more and more researchers try to exploit the non-linear mapping between channels either at partial or all RIS elements and to implement effective channel compression over the antenna space. Due to the universal approximation ability of the neural networks, deep learning (DL)-based channel extrapolation frameworks have been designed to infer the full channels from the partial ones over the antenna domain. The authors in [7] constructed a convolutional neural network (CNN)-based framework to complete the channel extrapolation over the antenna domain. Gao et al. developed a three-stage training strategy and utilize both fully connected network and CNN to realize the extrapolation task in [8].

However, in practice, the users can move and the channels between RIS and users would vary in time. As is well known, the higher the mobility speed is, the lower the channel coherence time is. Then, it would be more challenging to acquire a large number of unknown RIS channels within a limited channel coherence time. In this scenario, to ensure the system's spectrum efficiency, we will utilize as few pilot symbols as possible to achieve partial channel information within a given time interval. Hence, the idea of the channel extrapolation over the antenna domain could be applied for time-domain as well.

Thus, in this paper, we consider the time-varying cascaded channel estimation over RIS-assisted communication. We resort to DL and utilize the idea of channel extrapolation in both antenna and time domains. Correspondingly, the entire neural network can be divided into two parts, i.e., the time-domain and the antenna-domain extrapolation networks. Specially, in the former, we merge the recurrent neural network (RNN) with the neural ordinary differential equations (ODE), which can accurately describe the dynamics of the RIS channels. In the latter, we utilize another function of the ODE, i.e., modifying the structure of neural networks, and design an enhanced feedforward neural network (FNN) to achieve better extrapolation performance. Then, we cascade the two networks and design a training scheme to jointly optimize them.

\section{System And Channel Model}

Let us consider a scenario where a base station (BS) communicates with a single antenna user equipment (UE) via RIS. The BS's $M$ antennas are in the form of uniform linear array (ULA), and RIS is equipped with $N$ reflective elements in the form of a uniform planar array (UPA), including $N_{v}$ the 
vertical direction and $N_{h}$ in the horizontal direction. The links from the BS to UE include the direct link and the cascaded one via RIS. The cascaded link consists of the channel from BS to RIS and that from RIS to UE. Without loss of generality, as BS and RIS are placed at fixed positions with limited local scattering, the channel between them can be considered to be a light-of-sight (LoS) and time-invariant link under a long time interval and can be written as

$$
\mathbf{H}=\sqrt{M N} \alpha \mathbf{a}_{A}(\psi) \mathbf{a}_{R}^{\mathrm{H}}\left(\phi_{h}, \varphi_{h}\right) \in \mathbb{C}^{M \times N},
$$

where $\alpha$ is the complex channel gain. $\mathbf{a}_{R}(\phi, \varphi)$ and $\mathbf{a}_{A}^{\mathrm{H}}(\psi)$ denote the steering vectors of the RIS and BS, respectively, with $\phi$ and $\varphi$ as the azimuth angle and elevation angle of RIS, and $\psi_{h}$ as the angle of departure (AoD). $\mathbf{a}_{R}\left(\phi_{h}, \varphi_{h}\right)=\mathbf{a}_{e l}\left(\varphi_{h}\right) \otimes \mathbf{a}_{a z}\left(\phi_{h}, \varphi_{h}\right) \in \mathcal{C}^{N \times 1}, \mathbf{a}_{A}\left(\psi_{h}\right)=$ $\left[1, e^{j \frac{2 \pi}{\lambda} d \sin \psi_{h}}, \ldots, e^{j \frac{2 \pi}{\lambda} d(M-1) \sin \psi_{h}}\right]^{\mathrm{T}}$, where the $N_{v} \times 1$ vector $\mathbf{a}_{e l}\left(\varphi_{h}\right)=\left[1, e^{j 2 \pi \frac{d}{\lambda} \cos \varphi_{h}}, \ldots, e^{j 2 \pi \frac{d}{\lambda}\left(N_{v}-1\right) \cos \varphi_{h}}\right]^{\mathrm{T}}$ and the $N_{h} \times 1$ vector $\mathbf{a}_{a z}\left(\phi_{h}, \varphi_{h}\right)=$ $\left[1, e^{j 2 \pi \frac{d}{\lambda} \sin \phi_{h} \cos \varphi_{h, i}}, \ldots, e^{j 2 \pi \frac{d}{\lambda}\left(N_{h}-1\right) \sin \phi_{h} \cos \varphi_{h}}\right]^{\mathrm{T}} \cdot \quad \lambda$ is the carrier wavelength and $d$ denotes the antenna spacing. Furthermore, $\otimes$ is the Kronecker product operator and $[\cdot]^{\mathrm{T}}$ represents the transpose.

Due to the mobility of UE, the channel between RIS and UE experiences time-selective fading. Without loss of generality, we assume that the channel is quasi-static during a time block of $L_{c}$ channel uses and changes from block to block. Then, the value at the $n$-th time block is

$\mathbf{g}_{n}=\sqrt{\frac{N}{L_{g}}} \sum_{i=1}^{L_{g}} \beta_{i} e^{j 2 \pi\left(n \frac{v f}{c} \cos \theta_{i} L_{c} T_{s}-f \tau_{i}\right)} \mathbf{a}_{R}\left(\phi_{g, i}, \varphi_{g, i}\right) \in \mathbb{C}^{N \times 1}$,

where $\beta_{i}$ is the complex channel gain along the $i$-th path and $L_{g}$ is the number of scattering paths. $v, f, c$ and $T_{s}$ separately represent the moving speed of UE, the carrier frequency, the speed of light, and the system sampling period. $\tau_{i}$ and $\theta_{i}$ denote the time delay and the angle between the incident direction of the electromagnetic wave and the movement direction of UE for the $i$-th path.

Thus, at time $n^{\prime}$ of the $n$-th time block, the received signal at BS side is expressed as

$$
\mathbf{y}\left(n^{\prime}\right)=\mathbf{H} \boldsymbol{\Phi}\left(n^{\prime}\right) \mathbf{g}(n) s\left(n^{\prime}\right)+\mathbf{v}\left(n^{\prime}\right),
$$

where the diagonal matrix $\boldsymbol{\Phi}\left(n^{\prime}\right)$ denotes the amplitude and phase control information at RIS, i.e., $\boldsymbol{\Phi}\left(n^{\prime}\right)=$ $\operatorname{diag}\left\{\beta_{1}\left(n^{\prime}\right) e^{j \vartheta_{1}\left(n^{\prime}\right)}, \beta_{2}\left(n^{\prime}\right) e^{j \vartheta_{2}\left(n^{\prime}\right)}, \cdots, \beta_{N}\left(n^{\prime}\right) e^{j \vartheta_{N}\left(n^{\prime}\right)}\right\} \quad \in$ $\mathbb{C}^{N \times N}, s\left(n^{\prime}\right)$ is the user's transmitting data, and $\mathbf{v}\left(n^{\prime}\right)$ denotes the additive white Gaussian noise with zero-mean and variance $\sigma_{n}^{2}$. In order to represent the cascaded channel more clearly, the received signal can be written as

$$
\mathbf{y}\left(n^{\prime}\right)=\underbrace{\mathbf{H d i a g}\{\mathbf{g}(n)\}}_{\mathbf{C}(n)} \boldsymbol{\rho}\left(n^{\prime}\right) s\left(n^{\prime}\right)+\mathbf{v}\left(n^{\prime}\right),
$$

where $\mathbf{C}(n)$ is the cascaded channel of size $M \times N$, and the $N \times 1$ vector $\boldsymbol{\rho}\left(n^{\prime}\right)$ is formed by the diagonal elements of $\boldsymbol{\Phi}\left(n^{\prime}\right)$. We can obtain the vector $\mathbf{c}(n) \in \mathbb{C}^{M N \times 1}$ by vectorizing $\mathbf{C}(n)$. Notice that $n^{\prime}$ in $\mathbf{y}\left(n^{\prime}\right), \mathbf{v}\left(n^{\prime}\right), \boldsymbol{\Phi}\left(n^{\prime}\right), \boldsymbol{\rho}\left(n^{\prime}\right)$ and $s\left(n^{\prime}\right)$ denotes the instant time index, while $n$ in $\mathbf{C}(n)$, $\mathbf{G}(n)$ represents the block index.

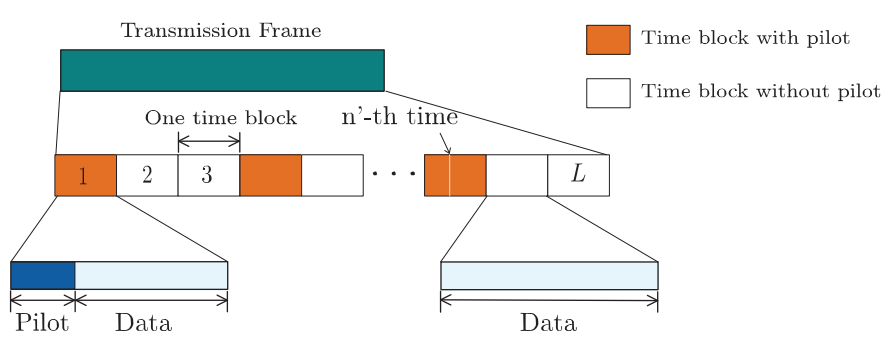

Fig. 1. The structure of the transmission frame.

\section{DL-BASED Channel Estimation Network}

\section{A. Proposed Problems}

We assume that each uplink frame from UE to BS contains $L$ time blocks as shown in Fig. 1. Before proceeding, let us define $\mathcal{L}=\{1,2, \ldots, L\}$ and $\mathcal{A}=\{1,2, \ldots, N\}$. To implement data detection, BS should recover $L$ matrices of size $M \times N$, i.e., $\{\mathbf{C}(n) \mid n \in \mathcal{L}\}$. In the RIS assisted communication with time-selective fading, the estimation of $\{\mathbf{C}(n) \mid n \in \mathcal{L}\}$ faces the following problem. At each time block, the size of the cascaded channel $\mathbf{C}(n)$ is proportional to the number of RIS elements, i.e., $N$. To acquire $\mathbf{C}(n)$, the length of the required pilot sequence is also proportional to $N$. In order to reduce this length, fewer RIS elements could be selected to implement the channel compression by controlling each RIS element's on/off state during each pilot block. Without loss of generality, we assume that the RIS elements during these pilot blocks have the same on/off pattern, and the indexes of all selected RIS elements are collected into the set $\mathcal{A}^{p}$, where $\left|\mathcal{A}^{p}\right|=N_{s} \ll N$. Correspondingly, the amplitude control information of the selected RIS elements in $\mathcal{A}^{p}$ is 1 , while for the others is 0 , i.e., $\beta_{i}\left(n^{\prime}\right)=1, i \in \mathcal{A}^{p}$ and $\beta_{j}\left(n^{\prime}\right)=0, j \notin \mathcal{A}^{p}$. Then, within each pilot block, our objective channel becomes matric of size $M \times N_{s}$, i.e., $\left\{\mathbf{C}_{:, \mathcal{A}^{p}}(n) \mid n \in \mathcal{L}\right\}$. Moreover, to ensure the system's spectrum efficiency, the number of pilot symbols should not be too large, which means that it is not necessary to insert pilot sequences in each time block. Define the set of time blocks for insertion of pilots as $\mathcal{L}^{p}$. Then, the $n$-th pilot block can be utilized to achieve the information about $\left\{\mathbf{C}_{:, \mathcal{A}^{p}}(n) \mid n \in \mathcal{L}^{p}\right\}$.

Due to the fixed RIS structure and channels' time correlation, the following mapping between $\left\{\mathbf{C}_{:, \mathcal{A}^{p}}(n) \mid n \in \mathcal{L}^{p}\right\}$ and $\{\mathbf{C}(n) \mid n \in \mathcal{L}\}$ exists

$$
\boldsymbol{\Phi}_{H}^{i}:\left\{\mathbf{C}_{:, \mathcal{A}^{p}}(n) \mid n \in \mathcal{L}^{p}\right\} \rightarrow\{\mathbf{C}(n) \mid n \in \mathcal{L}\}
$$

Since the spatial and time correlation of $\mathbf{c}(n)$ are uncoupled, the above mapping can be achieved through two sequential operations

$$
\begin{aligned}
& \boldsymbol{\Phi}_{H}^{t}:\left\{\mathbf{C}_{:, \mathcal{A}^{p}}(n) \mid n \in \mathcal{L}^{p}\right\} \rightarrow\left\{\mathbf{C}_{:, \mathcal{A}^{p}}(n) \mid n \in \mathcal{L}\right\}, \\
& \boldsymbol{\Phi}_{H}^{a}:\left\{\mathbf{C}_{:, \mathcal{A}^{p}}(n) \mid n \in \mathcal{L}\right\} \rightarrow\{\mathbf{C}(n) \mid n \in \mathcal{L}\},
\end{aligned}
$$

where the former denotes the channel interpolation along the time-dimension, while the latter is the channel extrapolation over the antenna-domain. 


\section{B. Initial Cascaded Channel Estimation}

In this part, we will resort to a simple linear estimator to achieve coarse information about $\left\{\mathbf{C}_{:, \mathcal{A}^{p}}(n) \mid n \in \mathcal{L}^{p}\right\}$. Without loss of generality, we assume that the pilot symbol from the user at the $i$-th time block is $\sqrt{\frac{P}{N^{p}}}$, where $i \in \mathcal{L}^{p}, N^{p}$ is the length of pilot sequence and $P$ is the pilot power during this time block. Let us collect $N^{p}$ observation vectors of size $M \times 1$ during the $i$-th time block inserted into $M \times N^{p}$ matrix $\mathbf{Y}_{i}^{p}=$ $\left[\mathbf{y}\left(n_{i}^{s}\right), \mathbf{y}\left(n_{i}^{s}+1\right), \ldots, \mathbf{y}\left(n_{i}^{s}+N^{p}-1\right)\right]$, where $n_{i}^{s}$ denotes the left border of the pilot sequence in the $i$-th time block, $i \in \mathcal{L}^{p}$. Their corresponding $N^{p}$ control vectors of size $\left|\mathcal{A}^{p}\right| \times 1$ are placed into the $|\mathcal{A}| \times N^{p}$ matrix $\boldsymbol{\Gamma}_{i}=\left[\boldsymbol{\rho}_{\mathcal{A}^{p}}\left(n_{i}^{s}\right), \boldsymbol{\rho}_{\mathcal{A}^{p}}\left(n_{i}^{s}+\right.\right.$ $\left.1), \ldots, \boldsymbol{\rho}_{\mathcal{A}^{p}}\left(n_{i}^{s}+N^{p}-1\right)\right]$. Then, with $\left[4, \mathbf{Y}_{i}^{p}\right.$ can be written as

$$
\mathbf{Y}_{i}^{p}=\sqrt{\frac{P}{N^{p}}} \mathbf{C}_{:, \mathcal{A}^{p}}\left(n_{i}^{s}\right) \boldsymbol{\Gamma}_{i}+\mathbf{V}_{i}^{p},
$$

where the $M \times N^{p}$ matrix $\mathbf{V}_{i}^{p}=\left[\mathbf{v}\left(n_{i}^{s}\right), \mathbf{v}\left(n_{i}^{s}+1\right), \ldots, \mathbf{v}\left(n_{i}^{s}+\right.\right.$ $\left.\left.N^{p}-1\right)\right]$. Let $\boldsymbol{\Gamma}_{i} \boldsymbol{\Gamma}^{H}=\mathbf{I}_{|\mathcal{A}|}$ and $N^{p} \geq|\mathcal{A}|$. Then, we can achieve the coarse estimation of $\mathbf{C}_{:, \mathcal{A}}(i)$ as

$$
\overline{\mathbf{C}}_{:, \mathcal{A}^{p}}(i)=\sqrt{\frac{N^{P}}{P}} \mathbf{Y}_{i}^{p} \boldsymbol{\Gamma}_{i}^{H}, \quad i \in \mathcal{L}^{p},
$$

where $\overline{\mathbf{C}}_{:, \mathcal{A}^{p}}(i)$ denotes the initial information of $\mathbf{C}_{:, \mathcal{A}^{p}}(i)$. Similar to the relation between $\mathbf{C}(i)$ and $\mathbf{c}(i)$, we define the corresponding vector version of $\overline{\mathbf{C}}_{:, \mathcal{A}^{p}}(i)$ as $\overline{\mathbf{c}}_{\mathcal{A}^{p}}(i)$. Then, we can achieve the coarse information about $\left\{\mathbf{C}_{:, \mathcal{A}^{p}}(i) \mid i \in \mathcal{L}^{p}\right\}$.

\section{DL-based Spatial Extrapolation and Temporal Interpola- tion for RIS Channels}

We first consider the mapping $\boldsymbol{\Phi}_{H}^{t}$ in 77 . Since RNN can effectively capture the time sequence's dynamical characteristics, we adopt it here. Before proceeding, let us define the raw input of RNN as $\mathbf{x}(n)$. Moreover, when $n \in \mathcal{L}^{p}$, $\mathbf{x}(n)=\overline{\mathbf{c}}_{\mathcal{A}^{p}}(n)$, and if $n \in \mathcal{L}-\mathcal{L}^{p}, \mathbf{x}(n)=\mathbf{0}$.

For a given time sequence set $\{\mathbf{x}(n) \mid n \in \mathcal{L}\}$, RNN would extract the hidden dynamical state set $\{\mathbf{u}(n) \mid n \in \mathcal{L}\}$. Hence, RNN-based channel interpolation contains two function blocks. The first one updates the hidden state $\mathbf{u}(n)$ utilizing RNN with parameters $\boldsymbol{\omega}_{R}$, which is denoted as "RNNCell $\boldsymbol{\omega}_{R}$ ". Here, $\mathbf{x}(n)$ is separately put into RNNCell $\boldsymbol{\omega}_{R}$ in the chronological order by taking $n$ from 1 to $L$. At each $n$, RNNCell $_{\boldsymbol{\omega}_{R}}$ deals with the current raw input $\mathbf{x}(n)$ and the previous hidden state $\mathbf{u}(n-1)$, and outputs the current state $\mathbf{u}(n)$. Correspondingly, the second block needs to infer $\mathbf{c}_{\mathcal{A}^{p}}(n)$ from the hidden state $\mathbf{u}(n)$, where a decoding network with parameters $\boldsymbol{\omega}_{D}\left(\operatorname{DecNet}_{\boldsymbol{\omega}_{D}}\right)$ is utilized. In fact, the output of $\operatorname{DecNet}_{\boldsymbol{\omega}_{D}}$ at time $n$ is the estimation of $\mathbf{c}_{\mathcal{A}^{p}}(n)$, i.e., $\widehat{\mathbf{c}}_{\mathcal{A}^{p}}(n)$. Then, RNN-based channel interpolation can be formulated as

$$
\left\{\begin{array}{l}
\mathbf{u}(n)=\operatorname{RNNCell}_{\boldsymbol{\omega}_{R}}(\mathbf{u}(n-1), \mathbf{x}(n)), \\
\widehat{\mathbf{c}}_{\mathcal{A}^{p}}(n)=\operatorname{DecNet}_{\boldsymbol{\omega}_{D}}(\mathbf{u}(n)), \quad n \in \mathcal{L} .
\end{array}\right.
$$

As we can observe from $10, \mathbf{u}(n)$ remains the same within the $n$-th and $(n-1)$-th time blocks, which does not fit the $\mathbf{c}_{\mathcal{A}^{p}}(n)$ 's dynamical characteristics, especially when the irregular pilot blocks are inserted. To deal with this problem, we resort to the ODE and model the dynamical hidden state

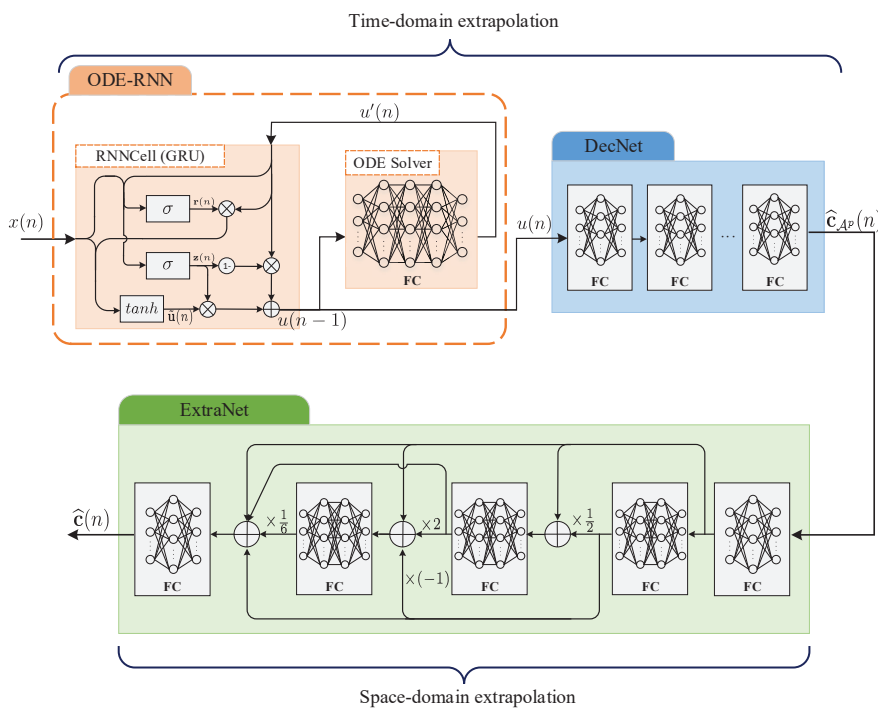

Fig. 2. The architecture of the proposed neural network for channel estimation.

$\mathbf{u}(n)$. Theoretically, ODE can be seen as a continuous-time model and can be formulated as

$$
\frac{d \xi(t)}{d t}=f(\xi(t), t)
$$

where $\xi(t)$ is the continuous dynamical state and $f(\cdot)$ specifies the dynamics of $\xi(t)$. In neural networks, $f(\cdot)$ can be approximated by a simple network with parameters $\boldsymbol{\omega}_{f}$. Then, $f(\cdot)$ can be written as $f_{\boldsymbol{\omega}_{f}}(\cdot)$. With a given $f_{\boldsymbol{\omega}_{f}}(\cdot)$ and the initial value $\xi(0)$, the numerical ODE solver, i.e., "ODESolver," can be utilized to evaluate the specific values of $\xi(t)$ at any desired time set $\left\{t_{0}, t_{1}, \ldots, t_{T-1}\right\}$ as

$\left\{\xi\left(t_{0}\right), \xi\left(t_{1}\right), \cdots, \xi\left(t_{T}\right)\right\}=\operatorname{ODESolver}\left(f_{\boldsymbol{\omega}_{f}}, \xi(0),\left\{t_{0}, \cdots, t_{T}\right\}\right)$.

Plugging ODESolver into (10, we formulate the ODE-RNN network structure, which can implement $\boldsymbol{\Phi}_{H}^{t}$ as

$$
\left\{\begin{array}{l}
\mathbf{u}^{\prime}(n)=\operatorname{ODESolver}\left(f_{\boldsymbol{\omega}_{f}}, \mathbf{u}(n-1),\left(t_{n}, t_{n-1}\right)\right), \\
\mathbf{u}(n)=\operatorname{RNNCell}_{\boldsymbol{\omega}_{R}}\left(\mathbf{u}^{\prime}(n), \mathbf{x}(n)\right), \\
\widehat{\mathbf{c}}_{\mathcal{A}^{p}}(n)=\operatorname{DecNet}_{\boldsymbol{\omega}_{D}}(\mathbf{u}(n)),
\end{array}\right.
$$

where $\mathbf{u}^{\prime}(n)$ is the middle hidden state output by ODESolver.

In particular, we adopt a fully connected neural network as the network of ODESolver and DecNet, and adopt the Gated Recurrent Unit (GRU) as hidden state update formula for the RNNCell function, which is defined as follows:

$$
\begin{aligned}
& \mathbf{r}(n)=\sigma\left(W_{r}\left(\left[\mathbf{u}^{\prime}(n), \mathbf{x}(n)\right]\right)\right), \quad \quad \text { reset gate } \\
& \mathbf{z}(n)=\sigma\left(W_{z}\left(\left[\mathbf{u}^{\prime}(n), \mathbf{x}(n)\right]\right)\right), \quad \text { update gate } \\
& \tilde{\mathbf{u}}(n)=\tanh \left(W_{\tilde{u}}\left(\left[\mathbf{r}(n) \odot \mathbf{u}^{\prime}(n), \mathbf{x}(n)\right]\right)\right), \quad \text { new memory } \\
& \mathbf{u}(n)=(1-\mathbf{z}(n)) \odot \mathbf{u}^{\prime}(n)+\mathbf{z}(n) \odot \tilde{\mathbf{u}}(n), \quad \text { hidden state }
\end{aligned}
$$

where $\sigma$ is the sigmoid function with the form of $\sigma(x)=$ $\frac{1}{1+e^{-x}}$, and $W_{r}(\cdot), W_{z}(\cdot)$ and $W_{\tilde{u}}(\cdot)$ are the network of reset gate, update gate, and new state update with different parameters, respectively. Moreover, $\mathbf{r}(n), \mathbf{z}(n)$ and $\tilde{\mathbf{u}}(n)$ are outputs 
TABLE I

Layer Parameters for the Proposed Model.

\begin{tabular}{|c|c|c|c|}
\hline & Layer & Output size & Activation \\
\hline ODESolver & $3 \times \mathrm{FC}$ layer & $M_{b} \times|\mathcal{L}| \times\left(M N \times 2 \times r_{a}\right)$ & Tanh \\
\hline $\begin{array}{c}\text { RNNCell } \\
\left(W_{r}(\cdot), W_{z}(\cdot), W_{\tilde{u}}(\cdot)\right)\end{array}$ & $6 \times \mathrm{FC}$ layer & $M_{b} \times|\mathcal{L}| \times\left(M N \times 2 \times r_{a}\right)$ & Tanh \\
\hline DecNet & $6 \times \mathrm{FC}$ layer & $M_{b} \times|\mathcal{L}| \times\left(M N \times 2 \times r_{a}\right)$ & Tanh \\
\hline ExtraNet & $8 \times \mathrm{FC}$ layer & $M_{b} \times|\mathcal{L}| \times(M N \times 2)$ & Tanh \\
\hline
\end{tabular}

of corresponding steps, and $[a, b]$ denotes the concatenation operation of $a$ and $b$.

Then, we consider the mapping $\boldsymbol{\Phi}_{H}^{a}$ in (7), which corresponds to the channel extrapolation process over the antennadomain. Similar to the super-resolution in the field of image processing, we can use the neural network to approximate this mapping from $\widehat{\mathbf{c}}_{\mathcal{A}^{p}}(n)$ to $\widehat{\mathbf{c}}(n)$. In the last task, ODE is employed to describe a dynamical changing process. In fact, according to the numerical solutions of ODE, we can modify the neural network structure and achieve better performance [9]. Here, we adopt a numerical solution of ODE, i.e., Runge-Kutta method, to modify the structure of feedforward neural network as the spatial extrapolation network [10]. Then, a network with parameters $\boldsymbol{\omega}_{E}$ called ExtraNet $\boldsymbol{\omega}_{E}$ can complete the following mapping as

$$
\widehat{\mathbf{c}}(n)=\operatorname{ExtraNet}_{\boldsymbol{\omega}_{E}}\left(\widehat{\mathbf{c}}_{\mathcal{A}^{p}}(n)\right) .
$$

Correspondingly, the proposed network architecture is shown in Fig. 2 Further, the detailed layer parameters settings we adopted in simulation are shown in TABLE [

\section{Learning Scheme}

As shown in the previous sub-section, the raw input of the neural network is $\{\mathbf{x}(n) \mid n \in \mathcal{L}\}$, and the total target is $\{\mathbf{c}(n) \mid n \in \mathcal{L}\}$. Moreover, as mentioned above, the network is divided into two parts: time-domain and antenna-domain extrapolation networks. Accordingly, in the network training stage, targets and loss functions should be set respectively for the two sub-networks to achieve the purpose of realizing different functions. Similarly, the target of time-domain extrapolation network, i.e., the ideal output of $\operatorname{DecNet}_{\boldsymbol{\omega}_{D}}$, is $\left\{\mathbf{c}_{\mathcal{A}^{p}}(n) \mid n \in \mathcal{L}\right\}$.

Let us define $\mathcal{T}$ as the network training dataset, where $|\mathcal{T}|=N_{t r}$ is the number of training sample. One sample in $\mathcal{T}$ contains three matrix sequences that are denoted as $\left(\{\mathbf{x}(n) \mid n \in \mathcal{L}\},\left\{\mathbf{c}_{\mathcal{A}^{p}}(n) \mid n \in \mathcal{L}\right\},\{\mathbf{c}(n) \mid n \in \mathcal{L}\}\right)$, where $\left\{\mathbf{c}_{\mathcal{A}^{p}}(n) \mid n \in \mathcal{L}\right\}$ and $\left.\{\mathbf{c}(n) \mid n \in \mathcal{L}\}\right)$ are the labels of timedomain and antenna-domain extrapolation networks, respectively. Without loss of generality, we use the mean square error (MSE) of the channel estimation as the loss function, which can be separately written as

$$
\begin{aligned}
\mathcal{L}_{t} & =\frac{1}{M_{b} M N_{s}|\mathcal{L}|} \sum_{i=1}^{M_{b}} \sum_{n \in \mathcal{L}}\left\|\mathbf{c}_{\mathcal{A}^{p}}(n)-\widehat{\mathbf{c}}_{\mathcal{A}^{p}}(n)\right\|_{F}^{2}, \\
\mathcal{L}_{a} & =\frac{1}{M_{b} M N|\mathcal{L}|} \sum_{i=1}^{M_{b}} \sum_{n \in \mathcal{L}}\|\mathbf{c}(n)-\widehat{\mathbf{c}}(n)\|_{F}^{2},
\end{aligned}
$$

where $\|\mathbf{A}\|_{F}$ is the Frobenius norm of matrix $\mathbf{A}$ and $M_{b}$ denotes the batch size for training. The total loss function is the weighted sum of $\mathcal{L}_{t}$ and $\mathcal{L}_{a}$, i.e., $\mathcal{L}_{s}=\mathcal{L}_{t}+\gamma \mathcal{L}_{a}$, where and $\gamma$ is the weighted coefficient. Here, the adaptive moment estimation (Adam) [11] algorithm is adopted to achieve the best network parameters $\boldsymbol{\omega}=\left\{\boldsymbol{\omega}_{f}, \boldsymbol{\omega}_{R}, \boldsymbol{\omega}_{D}, \boldsymbol{\omega}_{E}\right\}$, which is controlled by the learning rate $\eta$.

\section{Simulation Results}

In this section, we evaluate the performances of the proposed time interpolation and space extrapolation scheme through numerical simulation results. We first describe the communication scenario and the adopted dataset, and then introduce the parameter setting of the proposed neural network. Finally, the simulation results for performance evaluation are shown and explained.

We consider an environment with a BS, RIS, and UE. A BS equipped with two antennas $(M=2)$ communicates with the single antenna UE through RIS, which has 64 reflection elements $(N=64)$. It is assumed that the positions of BS and RIS are fixed, while UE can move at a high speed which is set as $100 \mathrm{~km} / \mathrm{h}$. The generation of the data is based on the DeepMIMO dataset [12], where the outdoor ray-tracing scenario ' $\mathrm{O} 1$ ' is adopted. The parameters $\alpha, \psi, \phi_{h}, \varphi_{h}$ can be extracted from the 'O1' scenario to generate the time-invariant channel $\mathbf{H}$. For generating the time-varying channel sample, we adopt the parameters $L_{g}, \beta_{i}, \phi_{g, i}, \varphi_{g, i}$ from DeepMIMO, and randomly select the angle between UE's movement and the direction of incident electromagnetic $\theta_{i}\left(\theta_{i} \in\left[-20^{\circ}, 20^{\circ}\right]\right)$. Then, we can generate the time-varying channel sample of each user according to (2). Furthermore, the carrier frequency of channel estimation is $28 \mathrm{GHz}$, and the system bandwidth is set as $20 \mathrm{MHz}$. The antenna spacing is $\frac{\lambda}{2}\left(d=\frac{\lambda}{2}\right)$ and the number of paths is set as $5\left(L_{g}=5\right)$. The time-domain sampling rate $r_{t}=\frac{\left|\mathcal{L}^{p}\right|}{|\mathcal{L}|}$ is set as a value in the set $\{0.3,0.5,1\}$, and the space-domain sampling rate $r_{a}=\frac{N_{s}}{N}$ is chosen in $\left\{\frac{1}{2}, \frac{1}{4}, \frac{1}{8}, \frac{1}{16}\right\}$.

The total number of cascaded channel samples is 20000 . We employ $80 \%$ of the data for training and the rest for testing. When calculating the loss function, we set the weight coefficient $\gamma$ as 1 to train the two networks jointly. The total number of epochs is 1000 , and the batch size $M_{b}$ is 200 . We set the initial learning rate $\eta$ as 0.005 , which decreases by $50 \%$ for every 50 epochs, and the lowest learning rate as 0.00005 .

Fig. 3 depicts the variation of normalized MSE (NMSE) versus epoch on the validation set at different spatial sampling rates. We adopt $\frac{1}{2}, \frac{1}{4}, \frac{1}{8}$ and $\frac{1}{16}$ sampling rates and consider the case of no noise. Obviously, it can be checked that the NMSE decreases with the epoch for all $r_{a}$. Further, it achieves the steady state within 1000 epochs, which proves the robustness of the proposed scheme. Moreover, with the increase of the number of RIS reflection elements, the performance of the proposed channel extrapolation scheme becomes better, converging to lower NMSE values.

Fig. 4 shows the channel extrapolation performance versus the antenna-domain sampling rate under different SNRs. The time-domain sampling rate $r_{t}$ is adopted as 0.3 . As can be seen from the figure, with the decrease of SNR at the same sampling 


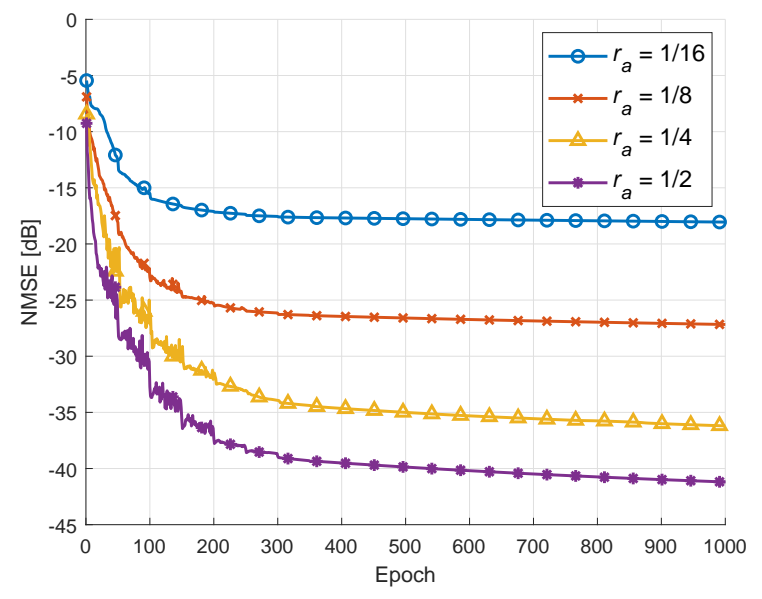

Fig. 3. NMSE of channel extrapolation vs. network training epoches for different $r_{a}$ values.

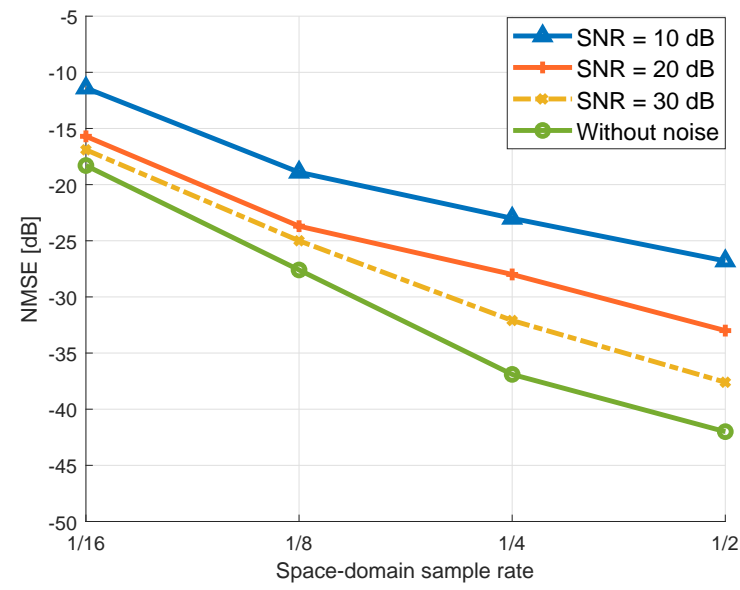

Fig. 4. NMSE of channel extrapolation vs. different antenna-domain sample rate for different SNR values.

rate, the NMSE gradually increases and the channel extrapolation performance gradually deteriorates. Furthermore, when noise is considered, the channel extrapolation performance becomes better with the increase of sampling rate, which is consistent with the results shown in Fig. 3

Fig. 5 describes the extrapolation performance of the designed network at both spatial and temporal sampling rates. We set SNR $20 \mathrm{~dB}$ and investigate the network performance under 4 different spatial sampling rates and 3 different temporal spatial sampling rates, i.e., $r_{a} \in\left\{\frac{1}{2}, \frac{1}{4}, \frac{1}{8}, \frac{1}{16}\right\}$ and $r_{t} \in\{0.3,0.5,1\}$. It is obvious that the NMSE decreases as the sampling rate increases, both for spatial and temporal sampling. This can be explained as the smaller the number of sampling points is, the less information about the cascaded channels is available, which is not conducive for the designed network to extrapolate the channel.

\section{CONCLUSION}

In this letter, we considered the time-varying channel acquisition problem in RIS scenario. To reduce the overhead of channel estimation, channel sub-sampling has been applied

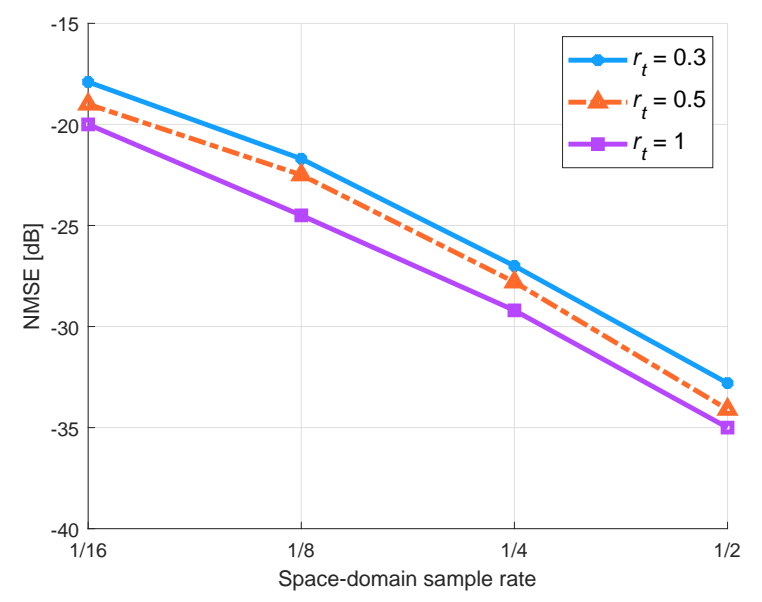

Fig. 5. NMSE of channel extrapolation vs. different space-domain sample rate for different $r_{t}$ values.

in both time and antenna domains, and a two-part cascaded neural network has been designed to accomplish channel interpolation in time-domain and channel extrapolation in antenna-domain through joint training. Furthermore, ODE has been utilized to describe the dynamic process of temporal interpolation in the former part of the network and promote the network structure in the latter, i.e., the antenna extrapolation part. Simulation results have illustrated that the cascaded channel extrapolation performance is satisfactory under the joint sampling of time and space domains, indicating that the proposed scheme is effective for time-varying channels and can also work well under the condition of noise, which proved its robustness.

\section{REFERENCES}

[1] Y. Han, W. Tang, S. Jin, C.-K. Wen, and X. Ma, "Large intelligent surface-assisted wireless communication exploiting statistical CSI, IEEE Trans. Veh. Technol., vol. 68, no. 8, pp. 8238-8242, Aug. 2019.

[2] C. Huang, A. Zappone, G. C. Alexandropoulos, M. Debbah, and C. Yuen, "Reconfigurable intelligent surfaces for energy efficiency in wireless communication, IEEE Trans. Wireless Commun., vol. 18, no. 8, pp. 4157-4170, Aug. 2019.

[3] Q. Wu and R. Zhang, "Towards smart and reconfigurable environment: Intelligent reflecting surface aided wireless network, IEEE Commun. Mag., vol. 58, no. 1, pp. 106-112, Jan. 2020.

[4] H. Liu, X. Yuan, and Y. -J. A. Zhang, "Matrix-calibration-based cascaded channel estimation for reconfigurable intelligent surface assisted multiuser MIMO," IEEE J. Sel. Areas Commun., vol. 38, no. 11, pp. 2621-2636, Nov. 2020.

[5] J. He, H. Wymeersch, and M. Juntti, "Channel estimation for RIS-aided mmWave MIMO systems via atomic norm minimization," IEEE Trans. Wireless Commun., pp. 1-1, Apr. 2021.

[6] S. Kim, H. Lee, J. Cha, S. Kim, J. Park, and J. Choi, "Practical channel estimation and phase shift design for intelligent reflecting surface empowered MIMO systems," arXiv:2104.14161, 2021, [Online]. Available: https://arxiv.org/abs/2104.14161.

[7] S. Zhang, S. Zhang, F. Gao, J. Ma, and O. A. Dobre, "Deep learning optimized sparse antenna activation for reconfigurable intelligent surface assisted communication," IEEE Trans. on Commun., pp.1-1, Jul. 2021.

[8] S. Gao, et al., "Deep multi-stage CSI acquisition for reconfigurable intelligent surface aided MIMO systems," arXiv:2104.11541 2021, [Online]. Available: https://arxiv.org/abs/2104.11541.

[9] R. T. Q. Chen, Y. Rubanova, J. Bettencourt, and D. Duvenaud, "Neural ordinary differential equations," arXiv:1806.07366v5, 2019, [Online]. Available: https://arxiv.org/abs/1806.07366v5. 
[10] M. Xu, S. Zhang, C. Zhong, J. Ma and O. A. Dobre, "Ordinary differential equation-based CNN for channel extrapolation over RISassisted communication," IEEE Commun. Lett., vol. 25, no. 6, pp. 19211925, Jun. 2021.

[11] O. P. Kingma and J. Ba, "ADAM: A method for stochastic optimization," arXiv:1412.6980 2014, [Online]. Available: https://arxiv.org/abs/1412.6980

[12] A. Alkhateeb, "DeepMIMO: A generic deep learning dataset for millimeter wave and massive MIMO applications," in Proc. Information Theory and Applications Workshop (ITA), San Diego, CA, pp. 1-8, Feb. 2019. 